Dans le cadre du «Forum Génériques» organisé à Zürich le 13 janvier dernier, le Président de la FMH a été appelé à apporter une contribution à un débat dont le titre était: «La liberté de contracter: une stratégie raisonnable pour la santé publique en Suisse?»; voici le texte de son intervention, légèrement remanié pour la publication.

\title{
«Liberté de contracter»: en est-il encore question?
}

\author{
Le temps des solutions - l'engagement des médecins
}

Jacques de Haller, Président de la FMH

\section{Qui donc sait ce qu'est la médecine?}

On a demandé à divers conférenciers de prendre la parole lors de ce Forum consacré à la pratique médicale: sept personnes au total, dont ... trois économistes et un seul médecin!

Tout est parfois fait comme si la médecine était un bien de consommation mis sur le marché par quelques entrepreneurs, visionnaires de génie, comme si la médecine était une prestation suffisamment définie par les sciences économiques.

Or la médecine n'est pas, et ne deviendra jamais, un «service» rentable et automatisé, une simple prestation qu'on pourrait chiffrer en deux clics de souris; il s'agit bien plutôt de l'accompagnement, millénaire, de celles et ceux qui souffrent, physiquement et ou psychiquement; il s'agit de la vie, du mieux-être et de la créativité de chaque habitant/habitante, de chaque personne vivant dans notre pays; il s'agit d'une science, d'un art, qui ne peut évidemment s'abstraire du monde dans lequel il s'exerce, mais qui ne doit en aucun cas être soumis à des règles qui le dénatureraient et qui le rendraient inopérant, qui en ferait une imposture - c'est un art délicat et fragile, que le corps médical est maintenant décidé à défendre de toutes ses forces, tant il croit à la valeur de ce qu'il fait.

On peut dire aussi, pour le représenter autrement ... et de façon plus polémique, mais ça n'est pas faux pour autant, que la médecine est manifestement à l'interface de deux facettes de la société, au point de contact entre ceux qui cherchent à façonner la société d'une part, et celles et ceux qui la vivent d'autre part.

Ce point de contact est celui où se produisent les étincelles: ça n'est pas par hasard que la médecine est devenue, dans le monde moderne, l'objet de demandes complètement contradictoires entre travailleurs, travailleuses et employeurs, entre assureurs et patients/patientes.

Vous comprendrez sans peine quelle importance a, pour nous, dans cette situation, une indépendance complète envers tous les protagonistes $d u$ système de santé - il n'est pas question d'irresponsabilité, mais bien d'indépendance: nous ne pouvons être soumis au diktat de qui que ce soit sans trahir une autre partie de notre vocation.

Ce qui n'arrange évidemment rien à la position de la Médecine dans notre monde qui s'essaie avec désespoir au néo-libéralisme, c'est qu'en elle-même, la médecine est une science molle, «soft», «fuzzy» si vous voulez, où l'important est souvent affectif - voilà qui n'est pas à la mode, et ça n'est guère conforme au modèle idéologique dominant.

Ça n'est guère conforme, c'est fortement suspect d'amateurisme, cela ouvre la porte à toutes sortes de suspicions, ... mais cela veut dire quelque chose quant aux priorités qui sont les nôtres; et là, ça redevient intéressant!

Il y a bien sûr des priorités qui sont spécifiques à l'une ou l'autre orientation de la médecine, qu'elle soit chirurgicale, centrée sur le psychisme, «sociale et préventive», ou que sais-je. Mais il y a des priorités qui nous sont communes, et qui sont si centrales qu'elles sont le squelette, la colonne vertébrale de toutes les spécialités médicales.

Soit dit en passant, c'est la redécouverte de ce fonds commun, de ce «fonds de famille», qui permet actuellement à la FMH de se reforger une unité, et c'est bien ainsi!

Ces priorités sont tellement constitutives de la pratique médicale qu'elles en acquièrent un caractère «obligatoire», pour nous: autant nous demandons avec force qu'elles soient prises en 
compte, que leur respect soit garanti par les divers acteurs du système de santé, autant elles nous engagent aussi nous-mêmes dans tout ce qu'elles supposent d'exigences dans l'exercice de notre profession.

On peut citer quatre de ces priorités, en commençant - vous ne serez pas surpris - par

- le fait de garder sa place centrale à la relation interpersonnelle entre le médecin et le patient, la patiente; cette relation est une donnée constitutive de la médecine, ça n'est pas une lubie de l'ère post-moderne; j'aime citer Hippocrate, pour montrer qu'à l'époque déjà, la relation entre le médecin et le/la malade pouvait dépasser la simple énumération de problèmes de fièvre ou de pustules: ne disait-il pas «Tu ne trahiras pas le secret de celui qui s'est confié à toi»? Comment mieux montrer qu'il y a 2500 ans déjà, aux origines de notre civilisation, la relation appartenait à la médecine?

Cette relation forte et si particulière appartient à notre métier, elle fait partie de la thérapeutique, elle doit absolument garder sa place et sa valeur, dans tous les sens du terme; elle doit garder son espace, qu'on peut appeler un «espace de créativité relationnelle», qui seul permet de bien soigner, et auquel nul n'a le droit de toucher, dans lequel nul n'a le droit d'intervenir - surtout pas en limitant le libre choix du médecin ...

- On doit citer ensuite, dans les priorités à mettre en exergue, la possibilité de préserver ce qu'on pourrait appeler notre «créativité thérapeutique»; cette créativité thérapeutique, c'est avec elle que nous, soignants/soignantes, sentons, dans un mélange subtil et nécessaire d'intuition, de connaissances, et de perception de l'autre dans son contexte, que nous sentons ce qui soignera, ce qui correspondra bien à la situation, ou ce qui, malgré toutes les lignes directrices du monde, ne collera pas à la réalité précise d'une situation.

Il y a donc, on l'aura compris, avant ou avec la liberté thérapeutique, la liberté de penser, la liberté de faire, la liberté de prescrire; non pas, bien sûr, la liberté de faire n'importe quoi, mais bien la liberté d'utiliser les instruments que nous avons pour détricoter une situation, puis pour lui trouver une solution; la liberté d'utiliser ces instruments avec bon sens mais aussi de manière créative.

On doit savoir que selon l'expérience vécue des médecins, les menaces qu'on fait peser sur notre avenir professionnel entravent notre capacité à penser librement, c'est-à-dire à penser sans contrainte et sans peur!
Et j'ai évoqué les lignes directrices: justement, elles doivent être cela, mais pas davantage, une indication, un guide, une direction, mais pas une recette de cuisine, et encore moins une norme obligatoire. On pourrait aussi évoquer ici le risque que feraient courir à notre créativité thérapeutique la limitation du catalogue des prestations, ou le risque pour les médecins d'être sélectionné(e)s, a fortiori sur la base de critères inadéquats, par exemple économiques.

- Autre priorité, non moins importante, celle de promouvoir et d'assurer la qualité.

La qualité des prestations d'une part, mais le sujet est trop vaste pour qu'on s'y étende ici: prestations techniques comme le laboratoire ou la radiologie, prestations invasives avec leur «outcome», traitement des patients chroniques avec le suivi de paramètres critiques taux de cholestérol ou tension artérielle obtenus sous traitement, pourcentage des visites chez l'ophtalmologue pour les diabétiques, etc., pour ne citer que quelques exemples.

Qualité des prestations d'une part, qualité du fonctionnement des cabinets d'autre part, qui est un domaine prometteur tant par l'amélioration des soins qu'il promet, que par les économies que l'on peut envisager par ce biais, qu'enfin par l'amélioration de la qualité de vie des praticiens/praticiennes. Plusieurs projets sont en route à ce sujet, dont le projet EPA auquel la FMH travaille actuellement.

La qualité des réseaux, formels ou informels, que les praticiens/praticiennes parviennent à constituer est aussi un élément de promotion de la qualité dans le fonctionnement des cabinets - les réseaux informels sont les réseaux que chaque praticien/praticienne constitue spontanément autour de sa pratique, le réseau des collègues avec lequels/ lesquelles on travaille régulièrement et auxquels/auxquelles ont sait pouvoir faire confiance, réseaux que l'on oublie trop souvent lorsqu'on se gargarise de thèmes à la mode en politique de santé...

- Parmi les priorités qui sont importantes pour nous, notamment pour structurer notre action politique alors que nous cherchons à échapper à l'emprise du tout-économique qui nous étouffe, parmi ces priorités importantes, la quatrième et dernière qu'il faut énoncer est celle, pour le corps médical, de rester ancré dans la vie sociale qui fait le quotidien des patients/patientes que nous voyons. Priorité importante s'il en est pour structurer notre action politique, car c'est une menace 
redoutable pour les médecins, depuis des siècles, que d'en rester à des modèles de société dépassés pour tout le monde sauf pour eux/elles, et de se couper ainsi de possibilités de dialogues et d'action combien plus positifs que le repli défensif.

Un exemple, à ce sujet: rester ancré dans la vie sociale de notre temps signifie, à une époque où plus personne n'achèterait un pain au chocolat ou une boite de conserves sans qu'y figure la totalité des ingrédients et une date de péremption, cela signifie qu'on ne peut plus vouloir faire de la médecine sans que nos «clients/clientes» puissent être assuré(e)s de la qualité de nos prestations et de notre formation - c'est normal, et nous devons nous y faire, alors que de rester drapé dans l'illusion que simplement parce qu'on est «Docteur», on est au-dessus de tout contrôle, n'est plus défendable.

L'information et «l'empowerment» des patients/patientes sont du même registre, du registre des réalités avec lesquelles on peut et doit vivre, comme l'économicité aussi face à des institutions - assureurs et, surtout, Etat qui assument une part essentielle des factures que nous établissons.

Notez bien que vivre avec une réalité actuelle et la prendre en compte ne veut pas dire qu'il faudrait tout accepter sans réagir, mais «simplement» qu'on ne peut pas, ou plus, refuser avec hauteur d'entrer en matière.

Bref, rester ancré dans la réalité sociale, ou plutôt, hélas, devoir s'y ré-ancrer, est une condition préalable à deux formes de dialogues auquel nous tenons tout particulièrement, à des niveaux différents: le dialogue avec nos partenaires du système de santé et, bien sûr, le dialogue avec les patients/patientes.

\section{La FMH et la révision de la LAMal}

Forte de ces convictions, la FMH a choisi l'option, nouvelle pour elle, de prendre le risque de positions publiques profilées, d'affirmer donc sa présence active dans le champ politique, et de faire en sorte qu'il ne se fasse plus de politique de santé sans la FMH, dans notre pays.

Nous avons ainsi estimé devoir faire des propositions concrètes dans le cadre du débat sur la révision - saucissonnée - de la LAMal; car le monde politique, se fondant certes sur des problèmes réels, mais aussi sur des interprétations au mieux discutables et au pire franchement inaccepta- bles, le monde politique est conduit à imaginer des solutions sur lesquelles il n'est malheureusement pas question pour nous d'entrer en matière.

Concrètement, dans la discussion sur le librechoix du médecin, les problèmes réels, ce sont ceux de garantir la qualité de la formation des médecins et des prestations fournies, et de l'information des patients/patientes à ce sujet, ainsi que de promouvoir l'économicité de l'activité médicale.

Ceci dit, il n'est simplement pas question, pour répondre à ces deux problèmes réels, de jeter aux orties le libre choix du médecin, et de renoncer du même coup à assurer l'emploi et les moyens d'existence de toutes celles et tous ceux de nos collègues qui travaillent bien - et c'est, on le sait bien, le cas de la quasi-totalité du corps médical; nous n'accepterons jamais de laisser une décision de vie ou de mort sur notre carrière dans les mains d'administrations qui ne sont pas toujours réputées ni pour la qualité de leur travail, ni pour la correction, la cohérence de leurs attitudes.

Nous proposons donc que l'automaticité de l'admission des médecins au remboursement de leurs factures soit gardée, préservant ainsi le corps médical de l'arbitraire d'une caisse-maladie ou d'une autre; cependant, pour répondre aux problèmes évoqués ci-dessus, nous suggérons que cette admission au remboursement des factures par les assurances sociales puisse être soumise à des critères supplémentaires, allant au-delà des critères basiques actuels (à savoir le diplôme de médecin et deux ans de stages):

Le Conseil fédéral peut faire dépendre l'admission des médecins de critères de qualité et d'économicité supplémentaires.

Il est évidemment indispensable que les médecins - les seuls qui savent ce qu'est la médecine! - élaborent eux-/elles-mêmes ces critères, le moment venu; personne d'autre ne devrait pouvoir nous les imposer. Mais pour assurer la crédibilité de la démarche et ne pas en faire une démonstration de corporatisme primaire, nous suggérons que ces critères ne soient pas élaborés par les seuls médecins, mais bien en commun avec les assureurs - assurément, le résultat d'une telle collaboration ne saura qu'être en même temps adéquat et efficace:

Le Conseil fédéral charge les partenaires tarifaires d'élaborer ces critères, uniformes sur le plan suisse, et il les approuve. 
Enfin, si médecins et assureurs n'arrivaient pas à se mettre d'accord, nous proposons que ce soit alors une commission fédérale ad hoc qui élabore des critères, commission constituée de représentants des médecins et des assureurs, mais aussi, pour arriver à un résultat positif et reconnu, de représentants/représentantes des cantons et des patients/patientes:

Si les partenaires tarifaires ne peuvent s'entendre sur ces critères, le Conseil fédéral les fixe. Pour ce faire, il s'appuie sur les propositions d'une commission paritaire.

La FMH pense proposer ainsi une solution qui répond efficacement et de manière crédible aux préoccupations qui sont derrière l'obsession actuelle de nos politiciens/politiciennes et des assureurs au sujet de la soi-disant «liberté de contracter», tout en parvenant à maintenir ce qui est pour nous une condition sine qua non, l'automatisme de l'admission au remboursement par les assurances sociales.

Nous ne saurions trop demander à nos divers partenaires de bien percevoir l'importance de ce projet de la FMH; il représente une vraie solution, et est un immense pas en avant de la part du corps médical, par rapport au passé, un pas en avant qu'il serait extrêmement regrettable de ne pas prendre au sérieux - tant il est vrai qu'un référendum pourrait ainsi être évité, référendum que nous aurions de très grandes chances de remporter mais qui par ailleurs n'apporterait rien à personne, car il ne ferait que nous ramener toutes et tous au point de départ!

Alors pour en revenir au titre de cet exposé, «Liberté de contracter: en est-il encore question?», ... eh bien, pour nous, la réponse est non!

Un «non» vigoureux, déterminé, solidement étayé par des convictions bien argumentées, et de surcroît un «non» constructif.

Le sous-titre de cette contribution était «Le temps des solutions - l'engagement des médecins»: vous avez entendu les propositions de la FMH; les médecins s'engagent ainsi de façon remarquable, et nouvelle, dans le champ politique, prêt(e)s à se construire un avenir d'ouverture et de créativité, un avenir où ils continueront à exercer un métier auquel ils et elles veulent croire avec confiance et enthousiasme. 